Stephan Kraft (Würzburg)

\title{
Gottfried Benn im „Rosen“-Dickicht. Über das im Briefwechsel mit F. W. Oelze am häufigsten erwähnte Gedicht
}

\begin{abstract}
Von keinem anderen Gedicht ist im Briefwechsel zwischen Benn und Oelze häufiger die Rede als von den in der Forschung bislang wenig beachteten „Rosen“. Tatsächlich handelt es sich bei dem Text aus dem Jahr 1946 um Benns Erstling nach der Schreibpause, die sich an das Kriegsende und den Suizid seiner zweiten Frau anschließt. Benn schwankt den „Rosen“ gegenüber offenbar selbst zwischen einer biographisch bedingten Anhänglichkeit und offen geäußerten ästhetischen Bedenken. Der Beitrag zeichnet das jahrelange, geradezu dramatische Hin und Her über Publikation oder Nichtpublikation, Widmen und Nichtwidmen zwischen Benn, Oelze und auch deren Ehefrauen Charlotte Oelze und Ilse Benn nach.
\end{abstract}

\section{1}

Das am häufigsten im Briefwechsel mit Oelze erwähnte Gedicht Gottfried Benns ist nicht „Orpheus’ Tod“ und auch nicht „Astern“, „Welle der Nacht“ oder „Die weissen Segel“. Anstelle all dieser viel berühmteren Texte wird diese Position von den vergleichsweise unscheinbaren „Rosen“ eingenommen: ${ }^{1}$

\footnotetext{
ROSEN

Wenn erst die Rosen verrinnen aus Vasen oder vom Strauch und ihr Entblättern beginnen, fallen die Tränen auch.

Traum von der Stunde Dauer, Wechsel und Wiederbeginn, Traum - vor der Tiefe der Trauer: blättern die Rosen hin.
}

\footnotetext{
1 Vgl. das Gesamtwerkregister in: Gottfried Benn, Friedrich Wilhelm Oelze: Briefwechsel, hg. v. Harald Steinhagen, Stephan Kraft und Holger Hof, Bd. 4: 1932-1956, Stuttgart und Göttingen 2016, S. 628. Im Folgenden wird auf diese Briefausgabe mit der Sigle „BOe I-IV“ im laufenden Text hingewiesen.
} 
Wahn von der Stunden Steigen

aller ins Auferstehn,

Wahn - vor dem Fallen, dem Schweigen:

wenn die Rosen vergehn. ${ }^{2}$

Die „Rosen“ sind nicht nur weniger populär als viele andere Gedichte, sie haben auch in der Benn-Forschung bislang kaum einmal eine eingehendere Betrachtung gefunden. Die Diskrepanz zwischen der häufigen Thematisierung durch Benn selbst, Oelze und - nicht zu vergessen - Charlotte Oelze und am Ende sogar noch Ilse Benn auf der einen und der weitgehenden Beiseitesetzung durch die Wissenschaft auf der anderen ist augenfällig. Eine Einzelinterpretation liegt nicht vor, ${ }^{3}$ und selbst in Hans Bryners Studie zum „Rosenmotiv in Gottfried Benns Lyrik“ findet man nur pflichtschuldige und recht unentschlossene fünf Seiten zu diesem Text. ${ }^{4}$ Zahlreiche Gedichte, in denen diese Blumenart nur relativ peripher auftaucht, werden von ihm wesentlich ausführlicher behandelt. Es scheint bei alldem eine gewisse Unsicherheit zu herrschen, wie mit diesem Text umzugehen ist.

Die „Rosen“ evozieren ganz zweifellos einen bestimmten, gut bekannten Benn-Sound. Hält man sie etwa neben die „Astern“ von 1936, so ist die Klangähnlichkeit verblüffend:

\author{
Astern - schwälende Tage, \\ alte Beschwörung, Bann, \\ die Götter halten die Waage \\ eine zögernde Stunde an. (SW I, 166) \\ Wenn erst die Rosen verrinnen \\ aus Vasen oder vom Strauch \\ und ihr Entblättern beginnen, \\ fallen die Tränen auch. (SW I, 227)
}

2 Gottfried Benn: Rosen, in: Ders.: Sämtliche Werke, Bd. I: Gedichte, hg. v. Gerhard Schuster, Stuttgart 1986, S. 227. Im Folgenden wird auf diese Werkausgabe mit der Sigle „SW I-VII/2“ im laufenden Text hingewiesen.

3 In einem Festschriftbeitrag von Jürgen Schröder finden sich drei Seiten, auf denen herausgearbeitet wird, dass das Gedicht partout nicht zur späten Lyriktheorie Benns passen will. Vgl. Jürgen Schröder: Gottfried Benns späte Lyrik und Lyriktheorie, in: Bild und Gedanke. Festschrift für Gerhart Baumann zum 60. Geburtstag, hg. v. Günter Schnitzler, München 1980, S. 410-424, hier: S. 413-415. Zwei Seiten sind es bei Anton Reininger, der darin vor allem ein ästhetisches Unbehagen vermittelt. Vgl. Anton Reininger: „Die Leere und das gezeichnete Ich“. Gottfried Benns Lyrik, Florenz 1989, S. 310-312.

4 Vgl. Hans Bryner: Das Rosenmotiv in Gottfried Benns Lyrik. Skizzen zu Bild und Bau, Bern u.a. 1985, S. 112-116. 
Auch erscheinen im Laufe des Textes neben den Rosen selbst zahlreiche weitere geläufige Benn-Motive: Traum, Schweigen, Steigen und Fallen, Ende und Wiederbeginn etc. pp. Man könnte das Gedicht angesichts dieser Massierung leicht für poetologisch bedeutend halten. Doch scheint ihm genau dies niemand so recht zuzutrauen. Vielmehr wirkt das Ganze wie eine Art Eigenpastiche. Der unter anderem aus den „Astern“ so gut bekannte Sound wird auf die Rosen als diejenige Blumenart appliziert, die eh am häufigsten in Benns Euvre vorkommt, und das Ganze wird dann durch eine Reihe von wohlbekannten Pathosvokabeln ergänzt. Mit etwas bösem Willen könnten man die „Rosen“ unter diesem Blickwinkel als eines der wohl erwartbarsten Gedichte Benns überhaupt bezeichnen.

Dafür, dass man sich nun trotzdem mit diesem Text beschäftigen sollte, der aus einer solchen, kritischen Perspektive wohl kaum eine Chance hat, in den engeren Kreis der vom Künstler anzustrebenden „hinterlassungsfähigen Gebilde“5 gerechnet zu werden, gibt es vor allem zwei Gründe.

Erstens beschäftigt sich Benn selbst sehr intensiv mit diesem Gedicht und kommentiert es vielfach, wobei ihn offenbar dieselben Zweifel plagen wie die spätere Forschung. Ist es ästhetisch gelungen, oder ist es einfach nur Kunsthandwerk? Gleichzeitig kann er von diesem Text aber auch nicht lassen. In der Folge entsteht zwischen ihm, Oelze und den jeweiligen Gattinnen eine höchst windungsreiche Diskussion um dieses Gedicht, die in der Folge nachgezeichnet werden soll.

Zweitens steht „Rosen“ an einem eminenten Punkt in der Biographie Benns. Üblicherweise setzt man „Orpheus’ Tod“ als den Neubeginn seines lyrischen Schaffens nach dem Ende des Zweiten Weltkriegs und dem Suizid seiner zweiten Frau Herta an. Das ist aber nicht korrekt, denn „Rosen“ wurde von Benn bereits drei Monate früher als „Orpheus’ Tod“ Anfang Juni 1946 auf den Weg zu den Oelzes nach Oberneuland geschickt. Übersehen wurde dies lange wegen der verzögerten Publikation, die erst 1949 in „Trunkene Flut“ erfolgte. Dieses Gedicht ist also - abgesehen von einem schlichten Widmungsvierzeiler ${ }^{6}$ - der eigentliche Nachkriegserstling Benns. Genau darauf gründet natürlich auch seine Anhänglichkeit an den Text, von dem er selbst zugleich nur zu gut weiß, dass er ein ästhetischer Problemfall ist, wie sich in der Folge noch zeigen wird.

5 Die Fügung erscheint bei Benn zunächst in „Züchtung“ (1940) (SW IV, 251) und „Julius Schmidhauser: ,Das Reich der Söhne““ (SW IV, 254) und wird dann noch häufiger wieder aufgegriffen. 6 „Sie standen bei der ,Morgue“ an meiner Seite“ (SW II, 131), für Rudolf Kurtz - wohl gedacht als persönliches Widmungsgedicht für die schließlich gescheiterte Henssel-Ausgabe der „Statischen Gedichte“ im Jahr 1946. 
Klaus Theweleit widmet im ersten Band seines „buchs der könige“ nicht weniger als 150 Seiten Gottfried Benn in der allerersten Nachkriegszeit ${ }^{7}$ und arbeitet dabei heraus, auf welche höchst problematische Art und Weise dieser nach dem Tod Hertas seine Lyrikproduktion wieder in Gang bringt. Dabei werden „Orpheus' Tod“ und das ebenfalls 1946 entstandene „Quartär“ detailliert kontextualisiert, um zu zeigen, wie Benn gerade aus dem Tod seiner Frau die Kraft zu neuer Kunst gesaugt habe. „Rosen“ wird hingegen irritierenderweise nicht ein einziges Mal erwähnt, obwohl Theweleit für seine 1989 erschienene Studie die Briefe Benns an Oelze mit dem gut sichtbaren „Rosen“-Gedicht an seinem korrekten historischen Ort im Mai/Juni 1946 bereits zugängig waren und auch häufig genutzt wurden. ${ }^{8}$

Auch in den sonstigen Orpheusinterpretationen spielen die in unmittelbarer zeitlicher Nähe entstandenen „Rosen“ kaum einmal eine Rolle. ${ }^{9}$ Es wird nicht einmal die ja durchaus vorhandene Möglichkeit genutzt, das Gedicht knapp als einen letztlich gescheiterten ersten Versuch eines lyrischen Neustarts zu fassen, bevor es dann mit „Orpheus’ Tod“ richtig losginge.

Immerhin wurde das Gedicht vor Kurzem an seinem historischen Entstehungsort gewürdigt. Thorsten Ries führt in seiner 2014 erschienenen genetischen Studie „Verwandlung als anthropologisches Motiv in der Lyrik Gottfried Benns“ die Gedichte „Rosen“, „Orpheus’ Tod“ und „Quartär“ als einen gemeinsamen Entwurfs- und Entstehungskomplex mit zahlreichen sich überkreuzenden Textlinien vor. ${ }^{10}$ Die Arbeitsschwerpunkte Benns ergeben dabei allerdings schon von sich aus, dass „Rosen“ erneut ein vergleichsweise geringes Interesse hervorruft.

7 Vgl. Klaus Theweleit: buch der könige, Bd. 1: orpheus und eurydike, Frankfurt a.M. 1989, S. 16-168. Der Argumentation Theweleits soll hier nur partiell gefolgt werden. Dass Benn aus dem Tod seiner Frau nachträglich künstlerisches Kapital geschlagen hat, ist schwerlich zu leugnen. Die weitergehende These, dass Hertas Tod von ihm dafür billigend in Kauf genommen oder sogar befördert wurde, halte ich allerdings für unzulässig spekulativ.

8 Prinzipiell bekannt war die Entstehungszeit auch schon früher. So wurde bereits im Kommentar der Benn-Werkausgabe von Dieter Wellershoff auf die Entstehung im Jahr 1946 hingewiesen. Vgl. Gottfried Benn: Gesammelte Werke in vier Bänden, hg. v. Dieter Wellershoff, Bd. 3: Gedichte, Wiesbaden 1960, S. 563. Vgl. auch Harald Steinhagen: Die statischen Gedichte von Gottfried Benn. Die Vollendung seiner expressionistischen Lyrik, Stuttgart 1969, S. 77.

9 Genannt seien beispielhaft zwei umfangreichere Interpretationsaufsätze: Renate Homann: Literatur als inhärente Komparatistik. Gottfried Benns Gedicht Orpheus’ Tod, in: Germanistik und Komparatistik, hg. v. Hendrik Birus, Stuttgart 1995, S. 92-110; Hugh Ridley: Orpheus Reborn Gottfried Benn's ,Orpheus' Death', in: Classics Ireland 3 (1996), S. 163-181.

10 Vgl. Thorsten Ries: Verwandlung als anthropologisches Motiv in der Lyrik Gottfried Benns. Textgenetische Edition ausgewählter Gedichte aus den Jahren 1935 bis 1953, Bd. 2, Berlin 2014 , S. 596-904. 
Der Produktionsprozess ist in diesem Fall einfach viel kürzer und kompakter als bei „Orpheus’ Tod“ und „Quartär“.

Die Arbeiten von Ries stellen eine notwendige Basis für das Folgende dar. Zeigen wird sich allerdings zugleich, dass sich das eigentliche Interesse hier weniger aus der Textgenese, sondern eher aus der weit darüber hinausreichenden, sehr dynamischen Kommunikation ergibt, die sich um dieses Gedicht herum entspinnt. Das „Rosen“-Drama ist eines, das erst durch eine Lektüre des vollständigen Briefwechsels in dieser Zeit überhaupt als ein solches erkennbar wird.

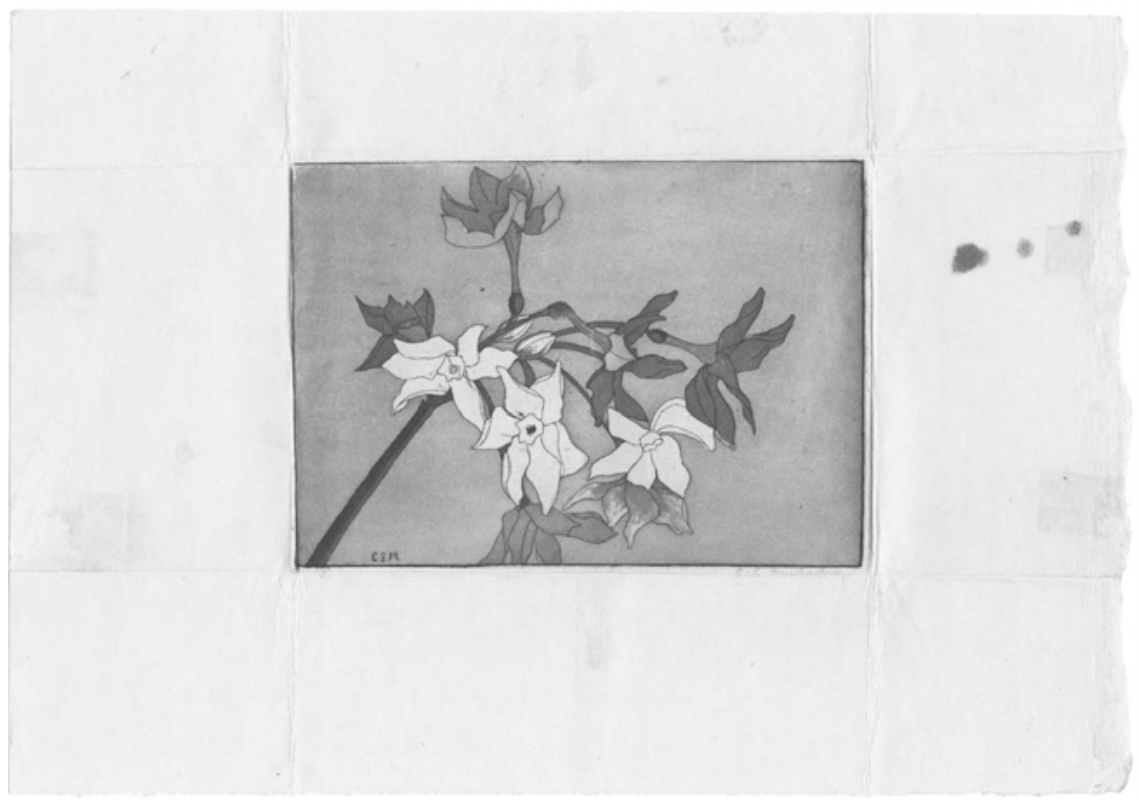

Abb. 1: Radierung Charlotte Oelzes, BOe II, 114; DLA Marbach

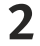

Am Anfang dieser Geschichte steht der Brief Oelzes vom 6. Januar 1946 (vgl. BOe II, 113-115), den er aus Mangel an Papier auf der Rückseite einer Radierung notiert, die seine Frau Charlotte in ihrer Jugend wahrscheinlich im Kunstunterricht einer Institution für höhere Töchter angefertigt hat. Oelze bemerkt dazu lakonisch: „Die Radierung ist von meiner Frau, als sie 18 Jahre war; jetzt Briefpapier.“ (BOe II, 115) 
Etwa um dieselbe Zeit, also Anfang 1946, legt Oelze einem seiner Schreiben eine Postkartenabbildung von dem Sommerhaus in Bremen-Oberneuland bei, in dem er und seine Frau seit Kriegsende leben, weil sie aus ihrem bisherigen Wohnhaus in Bremen-Horn ausquartiert worden sind. Das konkret verwendete Exemplar dieser Reproduktion einer Tuschezeichnung von unbekannter Hand ist nicht erhalten, doch wurden diese Postkarten auch in der Folgezeit von Oelze immer wieder einmal als Schreibgrundlage genutzt.

$\mathrm{Zu}$ diesen beiden optischen Eindrücken gesellt sich ein Themenkomplex, der davon zunächst ganz unabhängig ist. Ende Dezember 1945 (vgl. BOe II, 108) und dann nochmals Anfang März 1946 (vgl. BOe II, 126) öffnet sich Oelze erst- und einmalig in einem latent den Briefwechsel durchziehenden Punkt, nämlich seinem erotischen Interesse an jungen Männern. Benn fragt nach ersten Andeutungen des Bremers einmal irritiert nach (vgl. BOe II, 122) und ignoriert das Thema in der Folge von Oelzes Bestätigung vollständig. Es geht umgehend zurück zum üblichen ,Don't ask, don't tell.‘

Gleichsam in einer Gegenbewegung hierzu nutzt Benn die Radierung, die er als eine Abbildung von Christrosen identifiziert, sowie die Karte des Hauses in Oberneuland dazu, das Thema auf Charlotte Oelze zu lenken, die als Person im Briefwechsel der letzten 13 Jahre noch kaum einmal vorgekommen ist. Oelze hat diese private Seite seines Lebens bis dahin fast vollständig aus der Kommunikation mit Benn herausgehalten - und wenn doch, dann seine Familie immer nur ganz kurz und zumeist mit einem deutlich negativen Grundton erwähnt. ${ }^{11}$ Benn entschärft den aus seiner Sicht offenbar thematisch kritischen Moment Anfang 1946 also dadurch, dass er Oelzes Ehefrau als Gegenstand etabliert.

11 So etwa am 28. April 1935: „Da ich nichts von Ihnen hörte, blieb mir nur übrig, mich Ostern meiner Familie (im weiteren Sinne) zu ,widmen` - ein Zustand, den ich immer mehr fürchte, der mich jeder Haltung beraubt, - den ich fast schon empfinde wie einen Sturz ins trübe Element, in eine unwürdige Promiskuität ... schweigen wir davon, das Bittere würde überlaufen.“ (BOe I, 45) 


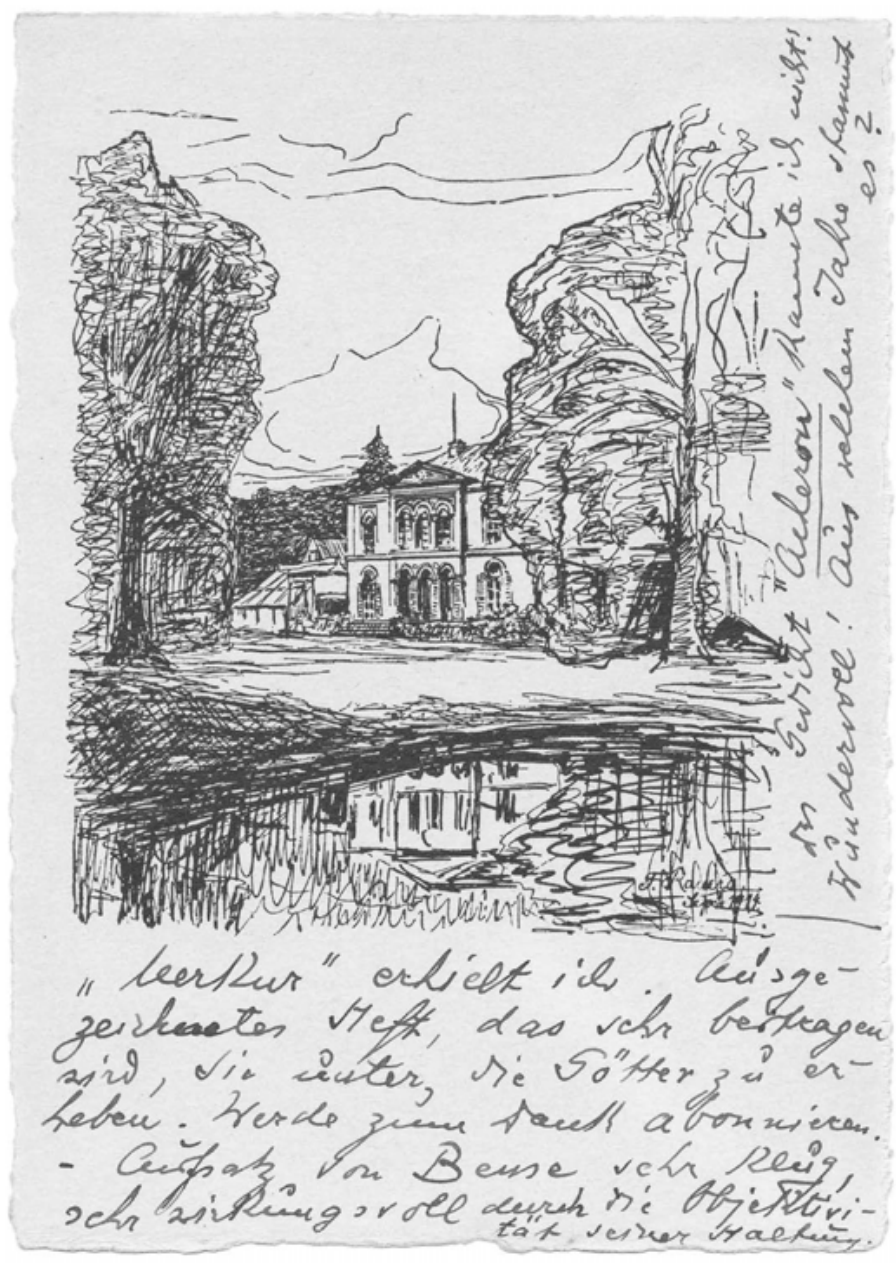

Abb. 2: Oelzes Wohnhaus in Oberneuland. Postkartenreproduktion einer Tuschezeichnung von unbekannter Hand, hier als Schreibgrundlage für Oelzes Brief vom 21. März 1949, B0e III, 51; DLA Marbach

Zugleich ist dies der Zeitpunkt, von dem an einzelne Schreiben zunächst von und an Charlotte Oelze und dann bald darauf auch Ilse Benn den Briefwechsel flankieren. Es werden im Viereck Grüße ausgerichtet und zu bestimmten Anlässen etwa zu Weihnachten oder zu Geburtstagen - auch Briefe an die Ehefrauen gerichtet bzw. von diesen verfasst. Dieser Teil der Kommunikation wirkt von außen betrachtet lediglich wie ein recht konventioneller Austausch von Höflichkeiten, ist es aber zumindest in der Genese keinesfalls. 
Benn setzt dieses neue Thema völlig übergangslos im unmittelbaren Anschluss an seine irritierte Nachfrage zu Oelzes erwähnten Geständnissen:

Ihr Haus in Oberneuland reizend, ganz Königin Luise. Übrigens die Christrosen Ihrer Gattin, die ich mir öfter betrachte, finde ich schön angeordnet u ausgestreut mit Phantasie. Sofern die Buchstaben links in der Ecke die Initialien des Mädchennamens sind, verwirrt es mich, ich dachte, Ihre Frau sei eine geborene Focke, (hat mir mal jemand erzählt.)? Menken? C. S. M? Verzeihen Sie, es ist nicht indiskret gemeint. (BOe II, 122)

Kurz darauf imaginiert er den Oberneuländer Garten als einen Rosengarten, der er natürlich zumindest zum Teil auch tatsächlich ist (vgl. BOe II, 143). Zur Rosenfrau gesellen sich die entsprechenden Beete. Damit ist das Rosenmotiv von dieser Seite her gleich doppelt gesetzt und unmittelbar etabliert.

Schaut man nun zeitlich parallel dazu in Benns Notizbücher, ${ }^{12}$ so steht dort die bereits Ende 1945 begonnene Arbeit an „Orpheus’ Tod“ im Zentrum. Bald erscheinen aber auch erste Notizen, die später in „Quartär“ eingehen werden. In „Quartär“ kommen dabei gar keine Blumen vor, in „Orpheus’ Tod“ immerhin ab dem späten Frühjahr 1946 der „gelbe Mohn“. ${ }^{13}$

Dann plötzlich taucht nur eine Seite weiter der Urentwurf von „Rosen“ auf ${ }^{14}$ und auf der Vorderseite des direkt folgenden Blattes gleich noch eine zweite Fassung, die mit der Datumsangabe „30/V“ versehen ist. ${ }^{15}$ Diese Notizen befinden sich aus dem Stand auf einer sehr weit fortgeschrittenen Stufe. Die ganze erste Strophe ist schon im primären Entwurf wortwörtlich diejenige des späteren Drucks, ${ }^{16}$ in den folgenden beiden wird noch ein wenig herumgeschoben und an den Details gefeilt. Es folgt dann eine Reihe von finalen Abschriften, ${ }^{17}$ von denen eine ebenfalls auf den 30. Mai datiert ist und nur zwei Tage später in die Post nach Oberneuland geht.

12 Im Zentrum steht hier das Notizbuch Nr. 11, das vor allem den Zeitraum vom Januar bis zum Juni 1946 abdeckt. Die für den vorliegenden Zweck relevanten Passagen finden sich reproduziert und aufgearbeitet bei Ries: Verwandlung [Anm. 10], S. 595-904.

$13 \mathrm{Nb}$ 11, Bl. 20r, zit. nach: Ries: Verwandlung [Anm. 10], S. 664-665.

14 Vgl. Nb 11, Bl. 21r, in: Ries: Verwandlung [Anm. 10], S. 668-669.

15 Vgl. Nb 11, Bl. 22r, in: Ries: Verwandlung [Anm. 10], S. 670-671. Steinhagen datiert die Entstehung ohne weitere Begründung auf den 4. und 5. Mai 1945. Vgl. Steinhagen: Die statischen Gedichte [Anm. 8], S. 77. In diesem Falle würde die Datierung auf der zweiten Fassung am ehesten wohl für den Zeitpunkt der recht wenigen Überarbeitungen stehen, die auf diesem Blatt zu finden sind.

16 Im Kommentarteil von SW I, 478, wird von Gerhard Schuster vermutet, dass diese erste Strophe als Widmung entworfen worden sei. Worauf sich diese Vermutung konkret gründet, bleibt offen.

17 Reproduktionen und Transkriptionen in: Ries: Verwandlung [Anm. 10], S. 678-685. 


\section{Riosen:}

Weun esst die Rusen verrinnen

lis rasen ader rom thanich

undich Entreattern heginnen,

palten dic Tränen arich.

Train in der stunden Dañer, beissel mad Wiederheginn,

Trainm- vir der Ticfe den Trames:

Geälien chi Risen him.

walm nin ar thimden Steigen

aller ins knipesteth,

Wahn - wor dem Fallen, dem Schrerifen:

nemn chi Risen vengehn.

$$
\text { S. B }
$$

Tor Frum Charlutte Kephanic oelze

und den farken in Obernenland.

$$
30 . \overline{1} 1946 \text {. }
$$

90.924

Abb. 3: Charlotte Oelze gewidmetes Manuskript der „Rosen“, DLA Marbach

Im Vergleich zu den höchst langwierigen Prozessen um „Orpheus’ Tod“ und „Quartär“ handelt es sich hier geradezu um eine lyrische Sturzgeburt. Unter dem Gedicht vermerkt Benn in der nach Oberneuland geschickten Endfassung: „Für Frau Charlotte Stephanie Oelze und den Garten in Oberneuland. 30. V 1946.“ (BOe II, 148)

Letztlich noch interessanter als die Widmung selbst, die kurioserweise ja nur zur Hälfte Oelzes Gattin und zur anderen Hälfte dem mit ihr assoziierten Garten gilt, als sei sie dessen ,Rosennymphe', ist der Text des separaten Begleitschreibens: 
Lieber Herr Oelze, ich denke öfter an Ihren Garten, in den Sie von den zwei Terrassenstufen aus steigen. Da ich ihn nicht malen kann $u$ keine Phantasie besitze, trachte ich ihn in einem kleinen Vers zu erfassen, den ich Ihrer Gattin, die ja nun auch schon soviele Jahre lang unbekannterweise mein Leben aus der Ferne begleitet, in ihre - wahrscheinlich wildlederbehandschuhten - Hände lege. Ein Dank wäre zuviel Belastung für diese kleine Melodie, - geschrieben auf den letzten Bogen des diesbezüglichen Papiers, das mich soviele Jahre begleitet hat. (BOe II, 147)

Dieser Brief wirkt bei genauerem Hinsehen vielleicht noch vielschichtiger als das Gedicht selbst. Oelze wird mit ihm gewissermaßen dafür getröstet, dass die „Rosen" an ihm vorbei seiner Gattin überreicht worden sind. ${ }^{18}$

Bemerkenswert sind an diesen drei kurzen Sätzen mehrere Dinge. Für das hier verfolgte Anliegen zentral ist natürlich die Bezeichnung der „Rosen“ als „kleine Melodie“, die man besser keiner „Belastung“ aussetzen sollte. Hierauf wird im Folgenden noch näher einzugehen sein. Vorab soll die Aufmerksamkeit aber noch auf zwei andere Dinge gelenkt werden.

Zunächst sind dies die „wahrscheinlich wildlederbehandschuhten - Hände“ Charlotte Oelzes. Damit ist sie natürlich nochmals als Rosenfrau, konkret als Rosenbewahrerin charakterisiert, die sich auf diese Weise vor den Stacheln ihrer Pfleglinge und eben auch dieses Gedichtes zu schützen suchen sollte.

Darüber hinaus dient dieses Attribut aber im Zusammenhang mit der zwischen dem Garten und ihr selbst zweigeteilten Widmung aber auch als Distanzmarkierung. Dieses Gedicht wird ihr eben nicht direkt in die Hände gegeben - es soll vielmehr noch eine trennende zweite Haut dazwischenliegen. Charlotte Oelze wird sich in der Folge als eine sensible Frau offenbaren, die derartige Botschaften durchaus versteht. Zugleich zeigt sich ihr praktischer Sinn. Sie lässt eigens eine Mappe aus blauem Maroquinleder anfertigen, in der das Manuskript separat einliegt (vgl. BOe III, 162). Charlotte Oelze nähert sich dem Gedicht, von dem sie später schreibt, dass es eines ihrer liebsten überhaupt sei (vgl. BOe III, 205), also nicht immer wieder selbst mit Handschuhen. Sie überzieht vielmehr den Text mit einer solchen separierenden Lederhülle. Deutlich wird hier auch, dass sich Charlotte Oelze dessen vollkommen bewusst ist, dass sie eben nicht die

18 Interessant ist, dass es im Briefwechsel keinen eindeutigen Hinweis darauf gibt, dass Benn und Oelze einander jemals Rosen geschenkt haben. (Zu der diesbezüglich missverständnisträchtigen Passage in BOe II, 328, vgl. den zugehörigen Kommentar BOe II, 535.) Andere Blumenarten schenkten sie einander sehr wohl, und Rosengeschenke an andere Männer kamen auch vor. Vgl. dazu etwa die Erwähnung eines entsprechenden Präsents von Benn an Rudolf Alexander Schröder in BOe IV, 67. 
wirkliche Empfängerin, sondern eigentlich nur die Treuhänderin dieses Textes ist. Auch das wird in der Folge noch eine wichtige Rolle spielen.

Dass die Gabenreihe, die mit der Rosenradierung Charlotte Oelzes unbewusst begonnen hat, nach dem „Rosen“-Gedicht noch eine höchst umständliche Fortsetzung findet, sei zumindest kurz angemerkt. Charlotte Oelze wählt als Gegengeschenk ein kleines Rosengemälde aus, das in der Folge vor allem deshalb noch mehrfach im Briefwechsel thematisiert wird, weil es sich in der Nachkriegszeit als sehr schwierig erweist, es von Bremen nach Berlin zu transportieren. Dies gelingt schließlich erst durch eine Botin im Jahr 1947. Das um 1800 entstandene, kleinformatige Bild wurde in Amsterdam erworben und ist nur über ein Foto aus dem Jahr 1953 bekannt, in dem es im Hintergrund an der Wand hängt. Es zeigt eine einzelne Blüte, die von Charlotte Oelze in einem nicht überlieferten ersten Dankesschreiben an Benn als Malmaisonrose angekündigt wird (vgl. BOe II, 160). Das kann nicht zutreffen, da diese konkrete Züchtung erst aus der Mitte des 19. Jahrhunderts stammt, doch handelt es sich um eine sehr ähnliche, vielblättrige und kugelförmige Blütenform.

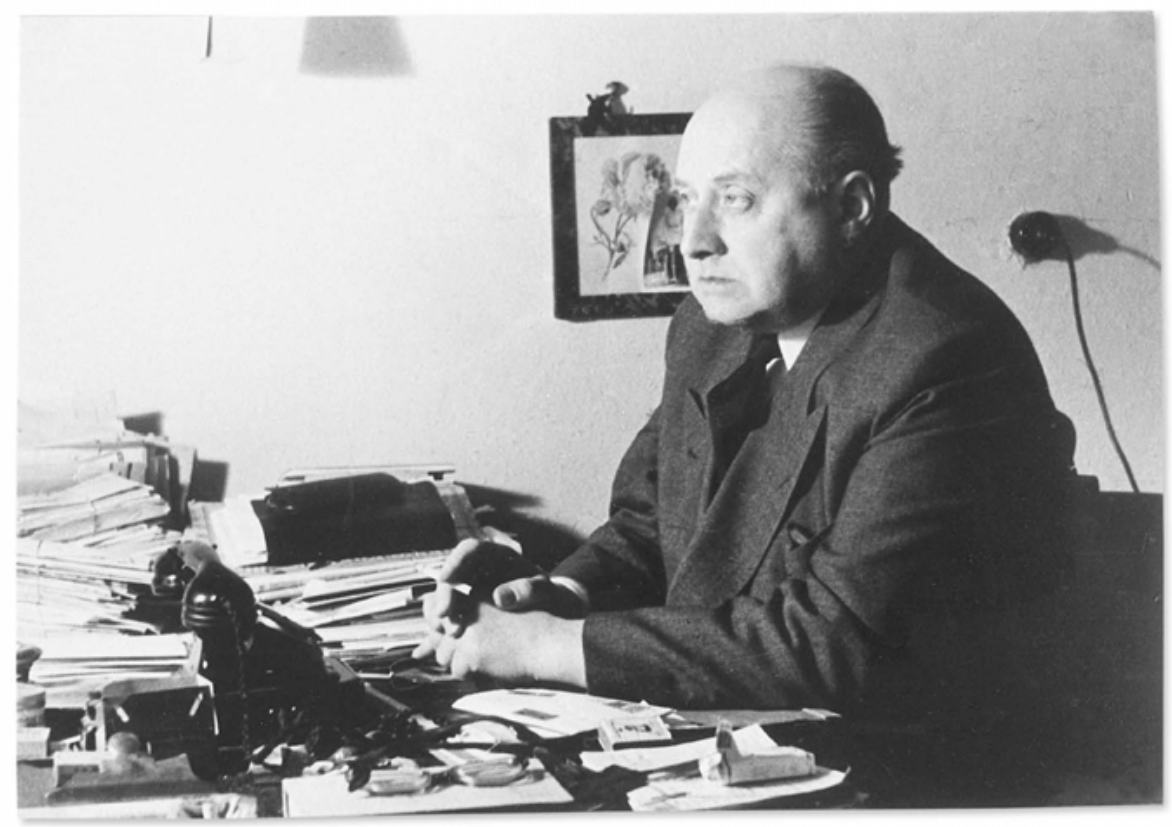

Abb. 4: Fotographie von Isa Ziesemer mit Benn an seinem Schreibtisch vor dem Rosenbild vom 5. Mai 1953; aus: Benn. Sein Leben in Bildern und Texten, hg. v. Holger Hof, Stuttgart 2007, S. 227; DLA Marbach 
Der zweite Punkt, der die Aufmerksamkeit in Benns Brieftext vom 1. Juni 1946 erregen kann, ist der Hinweis darauf, dass die Gedichtfassung, die er nach Oberneuland schickt, „auf den letzten Bogen des diesbezüglichen Papiers, das mich so viele Jahre begleitet hat“, notiert worden sei. Es handelt sich hierbei um ein cremeweißes Blankobüttenblatt im Format von etwa 16×22 cm, das unten eine auffällig breite und unregelmäßige Abrisskante aufweist. Wahrscheinlich war es wirklich das letzte Blatt eines Briefpapierblocks, das als solches an einer Schmalseite auf der üblicherweise zugehörigen Pappunterlage festgeklebt war. Neben dieser höchst pragmatischen Ebene, auf der sich Benn für den versehrten Zustand seiner Schreibgrundlage entschuldigt, kann diese Bemerkung aber auch darüberhinausgehend gedeutet werden. Benn ordnet implizit auch das auf dem Papier stehende Gedicht einer mit ihm selbst endenden Vergangenheit zu. Es ist gewissermaßen das letzte seiner Art.

Dass all das gut zu den zuvor angestellten Überlegungen zu den „Rosen“ als einem Eigenpastiche passt, sollte nachvollziehbar sein. Das Gedicht ist demnach primär der Nachklang einer eigentlich bereits untergegangenen Epoche. Wenn mit „Orpheus’ Tod“ zur selben Zeit ein neuer Ton für die Zukunft gesucht wird, dann tut Benn in der Folge in der Tat gut daran, die „Rosen“ aus diesem Komplex auszugliedern und an einem möglichst entlegenen Ort zu deponieren.

\section{3}

Zumindest für einen kurzen Moment geht die Verbindung von „Rosen“ und „Orpheus’ Tod“ dann aber doch noch über die reine zeitliche Koinzidenz hinaus. Auf der Vorderseite des auf die „Rosen“-Entwürfe direkt folgenden Blattes 23 desselben Notizbuches startet Benn einen Versuch, das Rosenthema in den Komplex von „Orpheus’ Tod“ zu integrieren:

halte Dich nicht mehr

hüten

Sinn

streue Dich, treibe die Blüten

Wie blätternde Rosen hin $^{19}$

Das „halte Dich nicht mehr“ stammt thematisch offenbar aus dem Kontext des Orpheusgedichts, die beiden unteren Verse sind aus dem der „Rosen“. Eine letzte

$19 \mathrm{Nb}$ 11, Bl. 23r, zit. nach: Ries: Verwandlung [Anm. 10], S. 674-675. 
Sicherheit gibt es zwar nicht, dass diese Zeilen aufeinander bezogen sind. Dafür spricht allerdings, dass die beiden Rosenverse hier weiter vom publizierten Endergebnis entfernt sind als die Entwurfsstufe ein Blatt zuvor. Vor allem aber ist das Rosenthema hier mit einer Ansprache an ein Du verbunden, das „Orpheus’ Tod“ grundiert, in den „Rosen“ aber weder in den beiden Entwürfen noch in der finalen Version jemals vorkommt. Es ist also davon auszugehen, dass es sich hier um einen, wenn auch recht kurzlebigen Versuch handelt, die beiden Komplexe miteinander $z u$ verbinden.

Zur Datierung ist anzumerken, dass sich auf derselben Seite der Entwurf eines Briefes an Oelze findet, mit dem Benn auf Oelzes Schreiben vom 30. Mai reagiert und den er am 10. Juni abschickt (vgl. BOe II, 149-151). Auch da sich schon auf der Rückseite von Blatt 22 eine Wendung notiert findet, die in denselben Brief eingeht, ${ }^{20}$ ist es sehr wahrscheinlich, dass der Versuch der Kombination der beiden Komplexe zeitlich nach dem Versenden des bereits fertigen „Rosen“-Gedichts nach Oberneuland liegt.

Wenn man nun - wie es sich etabliert hat - die Eurydike des Orpheusgedichts in einem Bezug zur verstorbenen Herta Benn sieht, erscheint dieser Versuch einer Verbindung der Komplexe durchaus plausibel, denn auch Herta ist für Benn zumindest zeitweise eine Rosenfrau gewesen. Verwiesen sei hier darauf, dass ihr Codename gegenüber Elinor Büller in den dreißiger Jahren „Rosenfinger“ gelautet hat. ${ }^{21}$ Die Verbindung Orpheus-Herta-Rosen ist also auch von daher durchaus eine mögliche, die von Benn dann aber schnell wieder gekappt wird.

Die „Rosen“ waren wohl der letzte der betörenden orphischen Gesänge, die keinen Platz mehr im neuen Kunstschaffen finden werden. Aber sind sie zumindest als solche gelungen? Oder ist hier das Gefühl der Trauer nach dem Verlust der Frau einfach zu unmittelbar und damit letztlich unkünstlerisch in ein Gedicht übersetzt worden. Ist dieser Nachklang mithin nur noch ein fernes Echo der alten Artistik und irgendwie ,Kunstgewerbe‘? Benn selbst kommt diese Befürchtung sehr bald. Mitte Juli notiert er sich einen Ausfall gegen seinen eigenen Text in sein Notizbuch, ${ }^{22}$ den er in seinem Brief an Oelze vom 19. Juli 1946 wiederholt und erweitert:

Ich sitze dann an einem bestimmten Fenster meiner Wohnung $\mathrm{u}$. schaue auf die leere $\mathrm{u}$. wenig begangene Strasse u sage mir, ein weiter Weg vom frühen G. B, dem wüsten Encephalitiker („Vermessungsdirigent“, „Karandasch“) bis zum Verfasser der harmlosen Rosen-

20 Vgl. Nb 11, Bl. 22v, in: Ries: Verwandlung [Anm. 10], S. 672-673.

21 Vgl. dazu Gottfried Benn: Briefe an Elinor Büller 1930-1937, hg. v. Marguerite Valerie Schlüter, Stuttgart 1992, S. 169 u. 173. Vgl. auch Theweleit: buch der könige [Anm. 7], S. 88-92.

22 Vgl. Nb 12, Bl. 2r, in: Ries: Verwandlung [Anm. 10], S. 690-691. 
verse, die von Gustav Falke sein könnten u von Phili Eulenburg komponiert -, zum Speien alles: das Stillestehn u. das Weitermüssen, der Stumpfsinn u. die Produktion, alles von Fratzen umstellt, von Zweifeln zerrüttet, von Schlagern an die Wand gedrückt u. aufgehoben. Ein weiter Weg, - ein alter Herr schliesslich das Resultat, der denkt, ob ihm jemand die Kragen plättet u. der auf die Strasse sieht. Hier ist Hochsommer. Brennnessel, Knöterich, Kraut wächst meterhoch auf dem Trottoir; (BOe II, 156)

„Brennnessel, Knöterich, Kraut“ - das sind die Pflanzen der Stunde. Rosen hingegen haben mit dem wahren Leben im zerstörten Berlin einfach nichts mehr zu schaffen. Sein Gedichtlein klingt Benn selbst wie süßlich-sentimentale Gartenlaubenlyrik..$^{23}$ Mit Philipp zu Eulenburg ${ }^{24}$ und Gustav Falke ${ }^{25}$ werden zielsicher zwei Vorgänger mit ähnlich musikaffinen, ja schlagerartigen Rosenversen aufgerufen.

Erkennbar wird hier, dass Benn nur zu gut weiß, dass er sowohl mit seinem Sound, seinen Reimen und vor allem mit seinen topisch verwendeten Blumenallusionen immer wieder am Rande des Kitsches balanciert. ${ }^{26}$ Für einen Fehltritt, wie er ihn hier selbst befürchtet, muss er gar nicht weit aus der Spur heraus.

Auf den ersten Blick scheint damit das schnelle Aus für die „Rosen“ unvermeidlich. Doch dem ist nicht so, wie ein Blick in die dichte Korrespondenz der Folgezeit zeigt. Mit dem Schreiben vom 31. August und 1. September schickt Benn „Orpheus’ Tod“ an Oelze. Dieser reagiert darauf am 7. September mit einem Brief, der Benn am 13. des Monats erreicht. Der genaue Blick auf die Daten ist nicht unwichtig, denn diese erste Reaktion aus Bremen kreuzt sich mit einem überraschenden Schreiben Benns vom 12. September:

Ich wage Sie um Ihr Urteil zu bitten, ob ich zu den „Statischen Gedichten“ „Rosen“ $u$. „Orpheus Tod“ hinzunehmen soll. (BOe II, 175)

23 In einem Brief an Max Niedermayer vom 14. Juni 1949 bezeichnet Benn „Rosen“ selbstkritisch als „Gartenlaubentitel“. Vgl. Gottfried Benn: Briefe an den Limes Verlag. 1948-1956, hg. v. Marguerite Valerie Schlüter und Holger Hof, Stuttgart 2006, S. 35.

24 Bezugspunkt sind hier die von Eulenburg auch selbst vertonten „Rosenlieder“. Dass mit der Nennung dieses Namens und der Evokation der Eulenburgaffäre um Kaiser Wilhelm II. auch das Thema der Homosexualität wieder in den Diskurs zurückkehrt, wird bereits in Ries: Verwandlung [Anm. 10], S. 900, Anm. 190, angemerkt.

25 Hier wären zahlreiche einschlägige Gedichttitel zu nennen, u.a. „Von weißen Rosen“, „Späte Rosen“, „Das Mädchen mit den Rosen“ oder „Rosentod“.

26 Vgl. auch Benns Schreiben an Ernst Jünger vom 24. April 1955: „Schade, dass, wenn man in einem Gedicht Geranien oder gar Rosen anbringt, die Experten sofort sagen: Vieu jeu, sentimental, 19. Jahrhundert. Nun, lassen wir sie.“ Gottfried Benn, Ernst Jünger: Briefwechsel 1949-1956, hg. v. Holger Hof, Stuttgart 2006, S. 51. 
Der konkrete Anlass zu dieser Reaktivierung der „Rosen“ ist eine neue Publikationshoffnung im Südverlag in Konstanz. Der Verleger gibt sich optimistisch, dass eine Veröffentlichung Benns in der französischen Zone gelingen könnte. ${ }^{27}$ Dass alte Verletzungen und eine beiderseitige Starrheit im Verhältnis zwischen Benn und Alfred Döblin, der in Baden-Baden als Zensuroffizier tätig ist, dem sehr wohl entgegenstehen werden, ahnen beide noch nicht.

Und auf einmal sind die „Rosen“ als Gegenstück zu „Orpheus’ Tod“ doch wieder im Spiel. Erneut sind die genauen Daten zentral. Benn scheint Oelze hier ehrlich um seinen Rat zu bitten, doch hat er zum einen erst gar nicht dessen Reaktion auf „Orpheus’ Tod“ abgewartet, und zum anderen hat er gegenüber dem Verlag bereits zuvor Nägel mit Köpfen gemacht. Das Manuskript der „Statischen Gedichte“, das er dem Südverlag fünf Tage zuvor zugeschickt hat, enthält nämlich beide Gedichte bereits. ${ }^{28}$ Was hier vorliegt, ist also letztlich nur die Simulation einer offenen Diskussion.

Das Ganze muss auf Oelze irritierend wirken. Da er aber natürlich nichts von Benns bereits erfolgter Integration der Gedichte in das aktuelle Konvolut wissen kann, überlegt er sehr ernsthaft, was sein Briefpartner wohl wollen mag:

- Was die Gedichtveröffentlichung betrifft, so erlaube ich mir, auf Ihre Frage zu erwidern, dass m. E. „Orpheus Tod“ durchaus in den Rahmen der Statischen Gedichte hineingehört [...], während das Rosengedicht in seiner melancholischen und zerbrechlichen Schönheit in diesem Rahmen vielleicht etwas fremd wirken könnte. (BOe II, 176)

Oelze übersetzt hier Benns wüste Eigeninvektive aus dem Brief vom 19. Juli zurück in den gemesseneren Ton des Begleitbriefs vom 1. Juni, in dem es ja noch hieß: „Ein Dank wäre zuviel Belastung für diese kleine Melodie“ (BOe II, 147).

Die Oppositionen dürften deutlich geworden sein: „Orpheus’ Tod“ ist von beiden Briefpartnern im näheren Umfeld mit dem Adjektiv „hart“ bezeichnet worden. ${ }^{29}$ Dieser Zug zur Härte ist offenbar die Richtung, die Benn mit der Hilfe

27 Vgl. den Brief von Johannes Weyl an Gottfried Benn vom 4. März 1946: „Die Intelligenz unserer Besatzungsmacht ist ausreichend für den Fall Benn, wenn es einen solchen gibt“. Zitiert nach Manfred Bosch: Zeit der schönen Not. Die Anfangsjahre des Südverlag in Konstanz 1945 bis 1952, Konstanz 2009, S. 324.

28 Vgl. zu diesem Manuskript Steinhagen: Die statischen Gedichte [Anm. 8], S. 75-77. Die dort abgedruckte Titelliste enthält auch „Orpheus’ Tod“ und „Rosen“. Dass genau dieses Konvolut an Weyl ging, ergibt sich aus einem unveröffentlichten Brief Benns an diesen vom 7. September 1946, der sich in Privatbesitz befindet.

29 Vgl. etwa Benn am 31. August / 1. September: „In der Skizze zu Orpheus sind die Dinge sehr hart neben einander gesetzt“ (BOe II, 170); ebenso Oelze am 28. September: ,wäre die Form nicht aus so hartem Material, wie es nur Ihnen zur Verfügung steht.“ (BOe II, 176) 
Oelzes nun gehen soll. Die „Statischen Gedichte“ sollen möglichst auch formal ,gepanzerte‘ Gedichte sein. Im Hintergrund dieses Wunsches steht natürlich zugleich genau das, was Theweleit als exklusives männliches Produktionspaar bezeichnet hat und das weite Teile des Jahres 1946 bestimmt habe. ${ }^{30}$ Dagegen sind die „Rosen“ bereits an sich eindeutig feminin konnotiert - und dann eben auch zart, zerbrechlich, nicht recht belastbar etc. pp. Es handelt sich hier um keine Männer-, sondern um eine gefährlich gefühlige Mädchenlyrik, von der sich der neu erstandene Benn nach Ansicht Oelzes besser trennen sollte. In Charlotte Oelzes schützendem Maroquinledermäppchen ist so etwas doch perfekt ver- und entsorgt.

Die Frage, ob Benn hier Oelze wirklich in einer Sache um Rat gebeten hat, die für ihn schon längst entschieden war, wird durch äußere Ereignisse gegenstandslos. In Konstanz und vor allem in Baden-Baden hat sich der Wind schnell und entschieden gegen eine Veröffentlichung von Texten Benns gedreht - ob nun mit oder ohne „Rosen“. 31

Für Benn ist diese erneute Niederlage bei den Versuchen, die „Statischen Gedichte" zu publizieren, ein Anlass, nach den zuletzt bemerkbaren Distanzierungsbewegungen doch wieder den Schulterschluss mit Oelze zu suchen. Im selben Schreiben vom 3. Oktober 1946, in dem er von dem Fehlschlag im Südwesten berichtet, tituliert er Oelze mit der berühmt gewordenen Formel als „Auftragsgeber u. Produktionsleiter“ (BOe II, 179). Das männliche Produktionspaar ist zumindest vorläufig restituiert, und tatsächlich verschwinden auch die „Rosen“ prompt und ohne dass darüber gesprochen würde wieder aus dem Konvolut.

In den überlieferten Titelaufstellungen der "Statischen Gedichte“ aus dem Jahr 1947 findet sich der Text im Gegensatz zu „Orpheus' Tod“ nicht wieder. ${ }^{32}$ Und auch in den dann endlich realisierten Ausgaben in der Schweiz und in Deutschland ist er nicht enthalten. Zugleich wurde die Sammlung aber um eine ganze Reihe von früheren Texten erweitert, da der Arche-Verleger Peter Schifferli gern eine größere Zahl von Gedichten für den Druck zusammenhaben wollte. Raum für die „Rosen“ hätte es also problemlos gegeben. Ein Kommentar Benns dazu ist nicht überliefert.

30 Vgl. zu diesem Konzept v.a. Theweleit: buch der könige [Anm. 7], S. 96-98.

31 Vgl. Bosch: Zeit der schönen Not [Anm. 27], S. 333-334.

32 Vgl. Steinhagen: Die Statischen Gedichte [Anm. 8], S. 80-81. 
Aber die „Rosen“ werden doch noch ihren Platz im veröffentlichten Werk Benns finden. Das Drama, das hier nachzuzeichnen ist, wird allerdings nicht mit einem grande finale, sondern eher als eine Parodie seiner selbst enden. Es folgt zum Schluss also ein sich langsam steigerndes Satyrspiel.

Mit dem Schreiben vom 14. Juni 1949 kündigt Benn Oelze im Zuge der Überlegungen, welche Texte nun in den großen lyrischen Rückblick „Trunkene Flut“ aufzunehmen wären, das Folgende an:

Ferner möchte ich das Gedicht Rosen (das mir im Laufe der Jahre infolge Ihrer etwas ablehnenden Kritik manches Kopfzerbrechen gemacht hatte) doch in den Band einfügen, vielleicht gegen Ende, etwa als 10 vor Schluss. Ich habe keine Abschrift der Aufstellung hier, die ich Ihnen schickte, und werde dementsprechend an N.[iedermayer] dazu Anweisung geben. Ich hänge an diesem kleinen melancholischen Gedicht, ich weiss nicht warum, vielleicht in Erinnerung an die Zeit seines Entstehens, des Moments seiner Konzeption, der Trauer, in der ich mich damals befand oder gottweiss warum. Wenn ich es mir heute ansehe, habe ich auch nur gegen die letzte etwas farblose und konventionelle Reihe etwas einzuwenden, sonst finde ich es zart und lyrisch. Ich habe auch daran gedacht, es unter die Epilog-Gedichte als etwa 3. einzusetzen, aber ich werde das doch wohl nicht tun, weil die Epiloggedichte stimmungsmässig in sich geschlossen sind, nämlich sehr persönlich und alle auf den Ich-ton eingestellt. Aber auch darüber würde mich Ihre Beurteilung interessieren, falls Sie für solche Kleinigkeiten Zeit und Sinn haben. (BOe III, 103)

Die Dinge wiederholen sich auf eine kuriose Weise: Wieder bildet eine sentimentale Grundstimmung die Basis, und wieder sollen die „Rosen“ nachträglich in eine Sammlung eingeschoben werden, ${ }^{33}$ in die das Gedicht nur halb passt. Schließlich versammelt die „Trunkene Flut“ mit Benngedichten vor allem der 20er und 30er Jahre bis auf das abschließende „Epilog 1949“ ansonsten ausschließlich bereits gedruckte Texte. Andersherum ließe sich aber - zweitens auch sagen, dass gerade dieser zeitliche Schwerpunkt als Umgebung für ein Gedicht sehr gut passt, dessen Rückwärtsgewandtheit zuvor schon mehrfach thematisiert wurde. Es schließt sich damit gewissermaßen ganz unauffällig an ,seinesgleichen' an. Zudem lässt sich ein solcher Problemfall nirgendwo besser sowohl zeigen als auch verstecken als irgendwo im hinteren Teil einer solchen Sammlung. ${ }^{34}$

33 In frühen Titellisten zu „Trunkene Flut“, die Teil des Briefwechsels mit Oelze sind, ist das Gedicht noch nicht enthalten. Vgl. BOe III, 66-67 u. 69.

34 Vgl. Gottfried Benn: Trunkene Flut. Ausgewählte Gedichte, Wiesbaden 1949, S. 109. Es ist damit das sechstletzte Gedicht im Band. Oelze schlägt zwar noch vor, dass „Rosen“ besser an die 
Das dritte Wiederholungsmoment besteht schließlich darin, dass erneut Oelze um Rat gebeten wird. Diesmal ist dies aber wohl wirklich gewünscht, wie aus einem Brief Benns an Max Niedermayer vom selben Datum deutlich wird:

Ein weiteres kleines ungedrucktes mit dem Gartenlaubentitel: „Rosen“, das ich anfänglich fortlassen wollte, an dem überlege ich noch. Ich erwarte noch Herrn Ö.s Äusserung dazu..$^{35}$

Oelze antwortet, nachdem Benn bereits am 20. Juni ungeduldig nachgefragt hatte (vgl. BOe III, 106), am 23. desselben Monats:

Was übrigens „Rosen“ betrifft, so habe ich dies Gedicht immer sehr zart und bezaubernd gefunden, weiss daher garnicht, wann ich es „ablehnend kritisiert“ hätte, - wie Sie in Ihrem Brief vom 14. VI. [...] schreiben. (BOe III, 108)

Benn hakt dann am 27. Juni nochmals nach, doch Oelze will sich auch in seiner Antwort vom 30. Juni partout nicht daran erinnern, sich jemals kritisch über die „Rosen“ geäußert zu haben. Und tatsächlich war es ja auch vor allem Benn selbst, der unzufrieden mit sich war und diesen Affekt nun auf Oelze verschiebt.

Noch im Mai 1950 - also lange nach der Publikation in der „Trunkenen Flut“ - fühlt sich Oelze bemüßigt, die schon wieder nach oben drängende „Rosen“Affäre im Detail richtigzustellen:

Regarding ,Rosen“ kann ich mich wohl insoweit exkulpieren, als ich lediglich von der Aufnahme dieses Gedichtes in die Sammlung der Statischen Gedichte abriet - weil es mir in dem durchgehenden maestoso dieser Gedichte allerdings zu lyrisch-zerbrechlich erschien -, das war keine Kritik an dem Gedicht als solchen, die Sie mir später unterstellten, und nur gegen diese Unterstellung, glaube ich, erhob ich bescheiden Einspruch; daß ich die Aufnahme in die Statischen widerraten hatte, das hatte ich nicht ,vergessen`. (BOe III, 290)

Irgendwie scheinen sich beide Protagonisten mit drei bis vier Jahren Abstand nicht mehr recht daran zu erinnern oder erinnern $\mathrm{zu}$ wollen, was sie damals in Sachen „Rosen“ getrieben haben oder vielmehr was sie getrieben hat.

Doch damit ist immer noch nicht genug: Auch im Zuge der Publikation gibt es noch einen kleinen, zweistufigen Nachklapp, der dem Ganzen nun endgültig einen Anflug von Slapstick verleiht. Im September 1949 sitzen Benn und Oelze parallel über den Fahnenkorrekturen für die „Trunkene Flut“, und beide stolpern

vorletzte Stelle direkt vor „Epilog 1949“ rücken sollte (vgl. BOe III, 181). Damit wären dann die unveröffentlichten Texte am Ende des Bandes beisammen. Benn legt diese Idee aber ohne größere inhaltliche Begründung ad acta (vgl. BOe III, 184).

35 Benn: Briefe an den Limes Verlag [Anm. 23], S. 35. 
über ein und denselben Namen. Benn schreibt am 22. September 1949 nach Bremen:

zu: Die weissen Segel -, würde ich Sie um Erlaubnis bitten, in diesem Band die Widmung an Sie fortzulassen, da ja das Rosengedicht Ihrer Gattin gewidmet ist und dann zweimal Oelze mir zu tautologisch erscheint. (BOe III, 163)

Das ist einmal mehr nur die halbe Wahrheit. Benn ärgert sich nämlich in erster Linie darüber, dass eine Abschrift des Typoskripts von „Rosen“ an den Verlag gelangt ist, die auch die Widmung an Charlotte Oelze enthalten hat. Es muss eine Spekulation bleiben, ob ihn nicht vielleicht weniger der Name als vielmehr die verräterische Jahreszahl 1946 gestört haben mag, die das Gedicht im aktuellen Kontext offen als nicht recht zugehörig ausweist. Am 20. August 1949 schreibt er an Niedermayer:

Unter uns gesagt: ich hatte eigentlich nicht die Absicht, das kleine Rosengedicht mit Widmung für Frau Oelze zu versehn, bin auch überrascht, dass es drunter steht. Offenbar hat Ihnen Herr O. ein Exemplar geschickt, das ich vor 4 oder 5 Jahren an ihn bzw seine Frau sandte, brieflich und ohne den Gedanken an eine Veröffentlichung. Aber nachdem das geschehn ist, kann ich es nicht gut ändern, es sei denn, Sie sagten mir, dass ich das Gedicht auch schon an Sie mit den anderen Manuskriptgedichten gesandt hätte, wo es nicht drauf stand. Dann könnte ich sagen, der Setzer habe dieses Exemplar gesetzt, auf dem keine Widmung stand. Aber das Ganze ist nicht so wichtig. Ich kenne Frau O. nicht, aber meine Frau, die jetzt einen Tag bei ihnen war, erzählt, dass es sich um eine sehr, sehr angenehme und kluge Dame handelt, der man schon höflich begegnen muss. ${ }^{36}$

Dass die Angelegenheit „nicht so wichtig“ ist, ist kaum glaubhaft, zumal Benn das Gedicht im Brief an Niedermayer erneut vom ,gefährlichen' Jahr 1946 wegrückt. Die erwähnten vier oder fünf Jahre Abstand würden vielmehr auf eine Entstehung in den Jahren 1944/45 hinweisen. Die Rosen sollen damit wohl eher im Kontext der späten Kriegs- als der frühen Nachkriegszeit verortet werden.

Nur ist ihm bei alldem die in der Tat „kluge Dame“ schon längst zuvorgekommen. Mit Benns Brief vom 22. September kreuzt sich ein Oelzebrief vom Vorabend, dem 21. September, 23.40 Uhr, in dem es heißt:

Meine Frau lässt gehorsamst bitten, die unter ,Rosen` (S. 85) stehende Widmung (die übrigens durch meine Abschrift s. Zt. nur versehentlich an Niedermayer gelangt ist) zu streichen, da sie sich unwürdig fühlt, mit ihrem Namen als einzige lebende Person vor der Weltöffentlichkeit, der Ihre Gedichte jetzt gehören, zu erscheinen. (BOe III, 162)

36 Benn: Briefe an den Limes Verlag [Anm. 23], S. 43. 
Jemanden mit einem Taktgefühl einer Frau Oelze muss man nicht erst bitten. Und auch Oelze zieht sich natürlich umgehend aus dem Band zurück. Benn erscheint geradezu beschämt. Sein Argument gegen die Doppelnennung ist irgendwie hinfällig und mit seiner ein wenig voreiligen Bitte räumt er die Oelzes etwas ruppig vollständig aus dem Buch heraus. Eine Geste soll die Situation offenbar retten. Im Brief vom 19. November des Jahres heißt es:

Schliesslich, bitte, Sie erhielten, wie mir N.[iedermayer] mitteilte, 2 Exemplare Trunkene Flut. Wollen Sie bitte in das eine Exemplar den beifolgenden Karton kleben und es Ihrer Gattin übergeben? (BOe III, 201)

Auf diesem Karton finden sich die folgenden Worte:

Frau Charlotte Oelze, der das Rosengedicht

S. 99 gewidmet war.

Gottfried Benn.

November 1949,

Berlin. (BOe III, 201)

Privat wird das öffentlich Getilgte als Geste für die Dame, „der man schon höflich begegnen muss“, dann also doch nochmals restituiert.

Soviel Geziehe und Gezerre gab es um kein anderes Gedicht Benns. Die Bedeutung dieser nur scheinbar belanglosen Kabalen geht dabei, wie gezeigt werden sollte, weit über das Privat-Biographische hinaus. Ohne die „Rosen“ ist die Krisengeschichte der Jahre 1945 und 1946 um „Orpheus’ Tod“ und „Quartär“ nur höchst unvollständig zu erzählen. „Rosen“ ist der lyrische Nachkriegserstling Benns, der als der letzte Nachklang der orphischen Gesänge alten Stils kein solcher sein und trotzdem nicht verschwinden durfte.

\section{5}

Und so ganz zu Ende ist die Geschichte selbst jetzt noch nicht. Mit Ilse Benn ist noch eine weitere Person einzubeziehen, die bei dem Verhandelten bislang nur am Rande aufgetaucht ist. Benn ist ihr praktisch unmittelbar nach der Fertigstellung der „Rosen“ und noch mitten in den Arbeiten zu „Orpheus’ Tod“ im Sommer 1946 erstmals begegnet. Man weiß, dass sie ihre Probleme mit den auf die Vergangenheit gerichteten Gedichten ihres Mannes hatte. Am 27. März 1949 schreibt dieser an Oelze: 
Doch in diesem einen Punkt ist sie [Ilse] schwierig, sie fühlt die innere u. äussere Macht, die meine verstorbene Frau immer noch über mich ausübt, und das kränkt sie. Daher auch Gedichte wie „Orpheus Tod“, „Dann“ - „Kleines süsses Gesicht“ von ihr bekämpft werden. (BOe III, 56)

Von den „Rosen“ ist hier zwar nicht die Rede, gemeint sind sie aber auch. Aktuell - also in den Jahren 1949 und 1950 - sind diese vor allem dank Oelze weiterhin höchst präsent. Beharrlich versucht er, Benn zu einem Besuch in Oberneuland zu bewegen und lockt neben der Rarität einer blühenden Kanadischen Felsenquitte, die es weit und breit nur in Oberneuland gebe, auch wiederholt mit den Rosen in seinem Garten (vgl. etwa BOe III, 294 u. 314). Auch vor dem Besuch Ilse Benns, die auf der Durchreise an die Nordsee im Sommer 1949 in Oberneuland Station gemacht hat, waren diese erneut Thema gewesen (vgl. BOe III, 124).

Und zu allem Überfluss verfasst Benn im Juni 1950 anstelle eines wiederum nicht stattgefundenen Besuches von ihm selbst in Bremen ein weiteres Rosengedicht für die verhinderte Gastgeberin Charlotte Oelze.

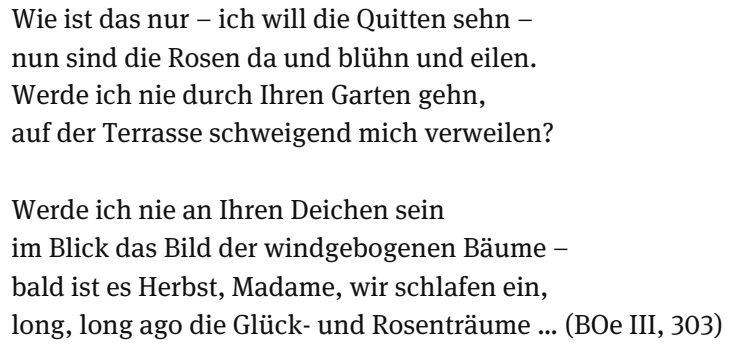

Ilse Benn zieht hier gewissermaßen die Notbremse. Am 4. Juli 1950 meldet ihr Gatte schließlich in Antwort auf einen weiteren Rosenbrief Oelzes:

Die Rosen, ja die Rosen! Meine Frau hat mir verboten, noch in einem Gedicht das Wort Rosen zu verwenden, - schade, es ist so ein schönes Wort. (BOe III, 315)

Hat sich Benn an diese Anweisung gehalten? Natürlich nicht. Selbstverständlich tauchen auch weiterhin Rosen in Benns Gedichten auf, so etwa in „Du übersiehst dich nicht mehr?“, „Spät, Keiner Weine -“, „Nur zwei Dinge“, „Viele Herbste“, „Destille“, „Tristesse“ oder „Letzter Frühling“. Gegenüber Astrid Claes klagt er 1954 über einen Sommer ohne Rosen. ${ }^{37}$ Selbst die Rosenkritikerin Ilse Benn be-

37 Vgl. Gottfried Benn: Briefe an Astrid Claes 1951-1956, hg. v. Bernd Witte, Stuttgart 2002, S. 39. 
kommt zum Hochzeitstag oder zum Jahrestag der ersten Begegnung gern diese Blumensorte überreicht. ${ }^{38}$

Und auch das „Rosen“-Gedicht gilt nach der Publikation nicht etwa als endgültig erledigt. Am 4. Oktober 1952 notiert sich Benn anlässlich eines Separatdrucks in einer Zeitung in sein Notizbuch: „In Frankf. Abendblatt ,Rosen““. Und im März 1956 - nur gut vier Monate vor seinem Tod - lässt sich Benn eine Vertonung der „Rosen“ durch den 1925 in Triest geborenen und 2011 ebenda gestorbenen Musiker Alessandro Mirt vorspielen. ${ }^{39}$

... und wenn man nun noch auf das berühmte Totenbild Benns im Sarg schaut, dann sind es eben auch dort just Rosen, die in seinen Händen liegen. Rosen und kein Ende.

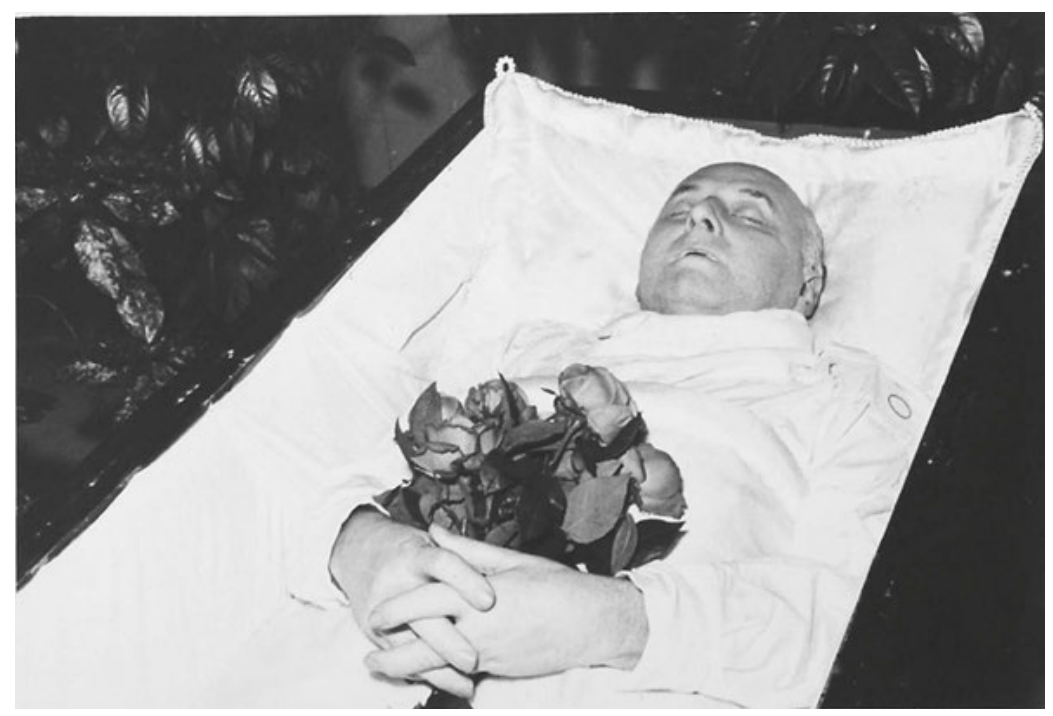

Abb. 5: Gottfried Benn im Sarg mit Rosen; aus: Benn. Sein Leben in Bildern und Texten, hg. v. Holger Hof, Stuttgart 2007, S. 269; DLA Marbach

38 Vgl. Benns Arbeitshefteinträge vom 18. Dezember 1953 und vom 27. Juli 1954 (AH 18a und AH 19c im DLA Marbach). Für diese und weitere Hinweise auf diverse Rosen in Benns bislang noch nicht publizierten Notizbüchern und auch anderswo sei Holger Hof sehr herzlich gedankt.

39 Vgl. Benns Notizbucheintrag vom 24. März 1956 (AH 22a im DLA Marbach). Vgl. den entsprechenden Eintrag in Helmut Heintel: Gottfried Benns Werk in der Musik. Eine Bibliographie der Vertonungen seiner Briefe, Gedichte, Prosa und Szenen, Stuttgart 1995, S. 69-70. 